Ann. Zootech., 1973, 22 (3), 267-277.

\title{
VALEUR PROTIDIQUE DES GRAINES DE VIGNA SINENSIS ET DE FÉVEROLE : COMPARAISON CHEZ LE RAT AVEC LE TOURTEAU DE SOJA
}

\author{
J. LE DIVIDICH \\ Station de Recherches zootechniques \\ Centre de Recherches agronomiques des Antilles et de la Guyane, I. N. R. A., \\ Domaine Duclos - Petit-Bourg - Guadeloupe
}

\section{RÉSUMÉ}

La valeur azotée des graines de Vigna Sinensis (variété M 53) et de Féverole (variété Ascott) a été estimée d'après un test de croissance, de digestibilité et de rétention azotée sur le Rat blanc, et comparée à celle du tourteau de soja cuit.

L'addition de o, I 5 p. Ioo de DL-méthionine aux graines de "Vigna Sinensis " et leur autoclavage à $120^{\circ}$ pendant 15 minutes entraînent un amélioration significative de la vitesse croissance, de l'efficacité alimentaire et de l'efficacité protidique.

En revanche, seule la supplémentation améliore significativement la vitesse de croissance des rats recevant les régimes à base de Féverole. Néanmoins, le traitement thermique accroît la digestibilité apparente de la matière organique et des protéines de la Féverole.

Dans nos conditions expérimentales, les graines de Vigna autoclavées et supplémentées, et de Féverole crue supplémentées par o, I5 p. Ioo de méthionine ont une valeur azotée voisine de celle du tourteau de soja non supplémenté.

\section{INTRODUCTION}

Les graines de légumineuses contiennent une quantité importante de matières azotées (20 à 40 p. IOo selon Morisson, I967). Elles représentent une source intéressante de protéines pour l'alimentation humaine et animale (AIKRoyd et Doughry, I964). Sur le plan qualitatif, leurs protéines présentent une teneur élevée en lysine ( 5 à $8 \mathrm{~g}$ pour $\mathrm{r} 6 \mathrm{~g}$ d'azote) et à ce point de vue, elles constituent un bon complément 
des céréales. Toutefois leur valeur biologique est limitée par un déficit important en acides aminés soufrés ( 1,2 à $3,0 \mathrm{~g}$ pour $16 \mathrm{~g}$ d'azote), et pour un certain nombre d'entre elles par la présence de substances toxiques thermolabiles de nature mal connue (Osborne et Mendel, igi7 ; Borchers et Ackerson, I950 ; Liener et Pallansch, I962).

Depuis ces dernières années, on assiste en Europe à un regain d'intérêt pour la culture des légumineuses à graines, de Féverole notamment, en vue du remplacement dans l'alimentation des monogastriques des produits azotés d'importation, par des aliments d'origine métropolitaine. Dans la région Caraibe, déficitaire également en protéines, la culture de l'espèce Vigna Sinensis, intéressante en raison de son rendement en grains sees (DERIEUX, I969), de conservation facile (POINTEL, I967), pourrait de la même façon permettre de réduire les importations de tourteaux.

A notre connaissance, il existe peu de données sur la valeur nutritionnelle des graines de Vigna Sinensis et les résultats concernant l'effet d'un traitement thermique sur la Féverole divergent selon les auteurs (BORCHERs et AckERSON,

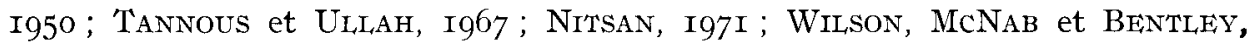
I972). Nous avons ainsi déterminé la valeur azotée des graines de Vigna Sinensis et de Féverole en comparaison avec le tourteau de soja, à l'aide d'un test de croissance, de digestibilité et de rétention azotée sur le Rat blanc. Les effets d'une supplémentation en DL,-méthionine de synthèse et d'un traitement thermique (autoclavage) ont également été étudiés.

\section{I. - MATÉRIEL E'T MÉTHODES}

\section{A. - Les graines et leur composition}

Les sources azotées étudiées sont constituées par un lot expérimental de graines de "Vigna Sinensis" (variété M 53) récolté en Guadeloupe (Station d'Amélioration des Plantes du C. R. A. A. G.), une Féverole de variété " Ascott " récoltée sur le domaine expérimental de La

TABLEAU I

Analyse des matières premières

\begin{tabular}{|c|c|c|}
\hline & Vigna Sinensis & Féverole \\
\hline Matière sèche $\ldots \ldots \ldots$ & 88,9 & $88,{ }^{\prime}$ \\
\hline Composition en p. 100 de la MS & & \\
\hline Matières azotées $(\mathrm{N} \times 6,25) \ldots \ldots \ldots$ & 27,5 & 32,0 \\
\hline Matières grasses $\ldots \ldots \ldots \ldots \ldots \ldots$ & 2,5 & 1,3 \\
\hline Cendres $\ldots \ldots \ldots \ldots \ldots \ldots \ldots \ldots$ & 3,5 & 3,9 \\
\hline Cellulose (Weende) ..... & 5,4 & 9,6 \\
\hline Extractif non azoté ........... & 61,1 & 53,2 \\
\hline Amidon vrai $\left({ }^{1}\right) \ldots \ldots \ldots \ldots \ldots$ & 39,1 & 36,8 \\
\hline
\end{tabular}

(1) Dosé par voie enzymatique à l'aide d'une amyloglucosidase (THIVEND et al, 1965). 
Minière (France) et un tourteau de Soja cuit à 44 p. Ioo de protéines. L'analyse chimique classique a été effectuée (tabl. I). Les teneurs en acides aminés indispensables sont estimées à partir des données de Busson (1965); Evans et Bandmer, (1967); PIoN et al., (1963) obtenues sur des échantillons de mêmes espèces végétales (tabl. 2)

\section{TABLEAU 2}

Calcul de la composition en quelques acides aminés indispensables

(p. roo de la matière sèche)

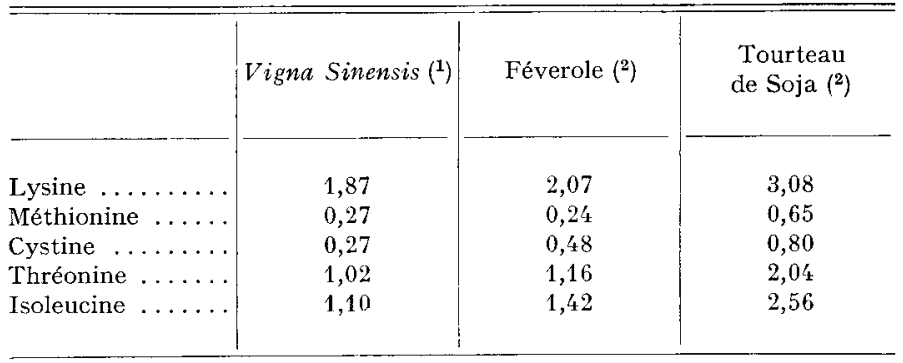

(1) D'après les données de Busson (1965) et Evans et Bandmer, 1967.

(2) D'après Pion et al., 1963.

\section{B. - Animaux, mise en lots, mode d'alimentation}

Soixante-quatre rats mâles, de souche Wistar ont été utilisés pour cette étude. A l'issue d'une période préexpérimentale d'une semaine après le sevrage, soixante sont répartis en Io lots comparables selon un dispositif factoriel (tabl 3) de 6 animaux chacun et quatre sont sacrifiés pour la détermination du contenu initial en azote corporel.

Les animaux sont placés en cages individuelles et reçoivent à volonté pendant une période de 12 jours, les régimes expérimentaux à sec (tabl. 3). Du $7^{\mathrm{e}}$ au $\mathrm{I}^{\mathrm{e}}$ jour de l'expérience, on a en outre récolté les fèces de 4 animaux par lot maintenus dans leur cage initiale, pour mesurer la đigestibilité apparente de la matière organique et l'azote des régimes.

A la fin de l'essai, tous les rats sont sacrifiés et congelés à - I $5^{\circ} \mathrm{C}$. Sur un échantillon homogène provenant du broyage de l'ensemble des carcasses d'un même lot, on a mesuré les teneurs en matière sèche, en cendre et en azote. La teneur en lipides est calculée d'après la différence : matière sèche - [protéines $(\mathrm{N} \times 6,25)+$ Cendres].

\section{C. — Nature des régimes}

Les farines de Vigna, de Féverole et le tourteau de soja constituent les seules sources azotées des régimes; leur taux d'incorporation est respectivement de 6o, 45 et $30 \mathrm{p}$. Ioo environ et la teneur correspondante des régimes en matière azotées est en moyenne de I4 p. Ioo. Les teneurs en cellulose n'ont toutefois pas été les mêmes d'un régime à l'autre : elles sont deux fois plus élevées avec la Féverole qu'avec le tourteau de Soja.

Les graines de Vigna et de Féverole sont autoclavées après broyage, en atmosphère humide à $120^{\circ}$ pendant 15 minutes (Evans et BANDMER, I967). Les régimes à base de Vigna, de Féverole et de tourteau de soja sont supplémentés en DL-méthionine à raison respectivement de $0,15,0,15$ et 0, Io p. IoO.

\section{D. - Critères de détermination de la valeur des protéines}

Les critères étudiés sont la vitesse de croissance, l'utilisation digestive apparente de l'azote, et la rétention azotée mesurée à partir de l'analyse chimique corporelle. Les principaux critères calculés sont les suivants : 
J. LE DIVIDICH

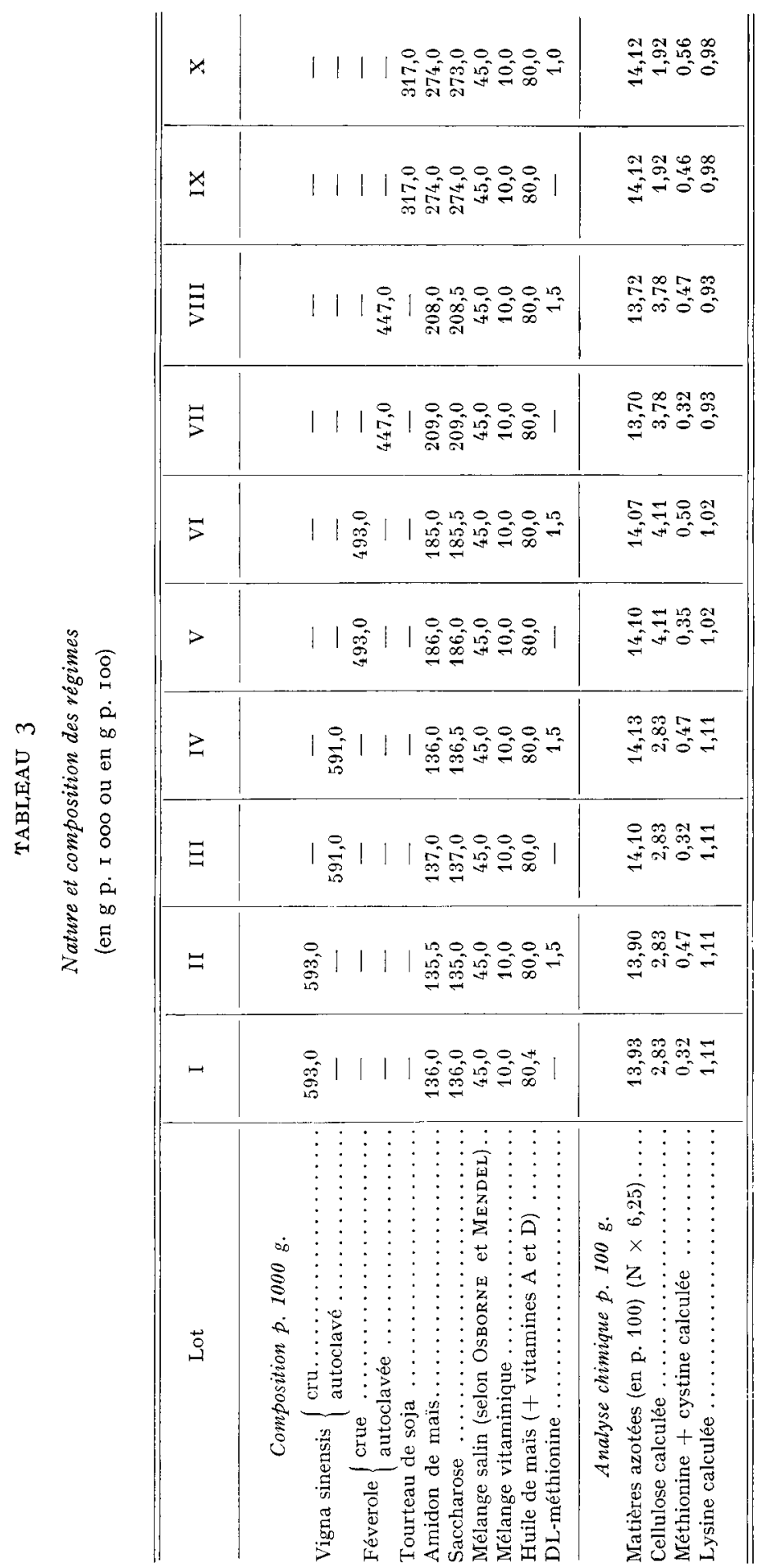


- Efficacité alimentaire :

- Coefficient d'utilisation digestive apparente de l'azote (CUD) ;

- Coefficient d'efficacité protidique (CEP) ;

- Coefficient d'utilisation pratique de l'azote (CUP) :

exprimés dans les unités habituelles.

\section{II. - RÉSULTATS}

\section{A. - Croissance et consommation d'aliments}

Les résultats de croissance et de consommation d'aliments sont rapportés dans le tableau 4 .

a) Infuence de la cuisson et de la supplémentation en DL-méthionine.

Dans le cas des régimes à base de Vigna, la cuisson et la supplémentation en DL-méthionine améliorent significativement la vitesse de croissance $(\mathrm{P}<0, \mathrm{OI})$ et la consommation journalière $(\mathrm{P}<0,05)$, des animaux. L'autoclavage et la supplémentation en DL-méthionine ont des effets additifs sur l'efficacité alimentaire et le coefficient d'efficacité protidique (CEP) et les performances maximales sont obtenues avec le régime cuit et supplémenté.

En revanche, dans le cas des régimes à base de Féverole, seule la supplémentation améliore significativement $(\mathrm{P}<\mathrm{o}, \mathrm{OI})$ l'ensemble des performances.

Pour ce qui concerne les régimes à base de tourteau de Soja cuit, on note un effet significatif de la supplémentation en méthionine sur la vitesse de croissance des animaux $(\mathrm{P}<0,05)$ et le CEP $(\mathrm{P} .<0,05)$.

b) Comparaison au tourteau de soja.

Le régime à base de tourteau de soja cuit non supplémenté est choisi comme terme de comparaison en raison de sa teneur en acides aminés soufrés (facteurs limitants) équivalente à celle des régimes à base de Vigna et de Féverole supplémentés.

Dans ces conditions, les performances de croissance enregistrées avec les régimes à base de Vigna cuit et supplémenté, et de tourteau de Soja cuit non supplémenté sont comparables et supérieures (de $7 \mathrm{p}$. I0o) à celles obtenues avec le régime à base de Féverole crue et supplémentée.

L'efficacité alimentaire et le CEP de ces mêmes régimes à base de Vigna et de tourteau de soja sont également comparables et supérieurs (de 8 p. Ioo) à ceux du régime à base de Féverole crue supplémentée.

\section{B. - Coefficient de digestibilité apparente}

En l'absence d'effet de la DL-méthionine supplémentaire sur l'utilisation digestive, il n'en est pas tenu compte ni dans la présentation, ni dans l'analyse des résultats (tabl. 5).

La cuisson accroît sensiblement la digestibilité apparente de la matière organique $(\mathrm{P}<0,05)$ des régimes à base de Vigna et de Féverole. Elle améliore nette- 


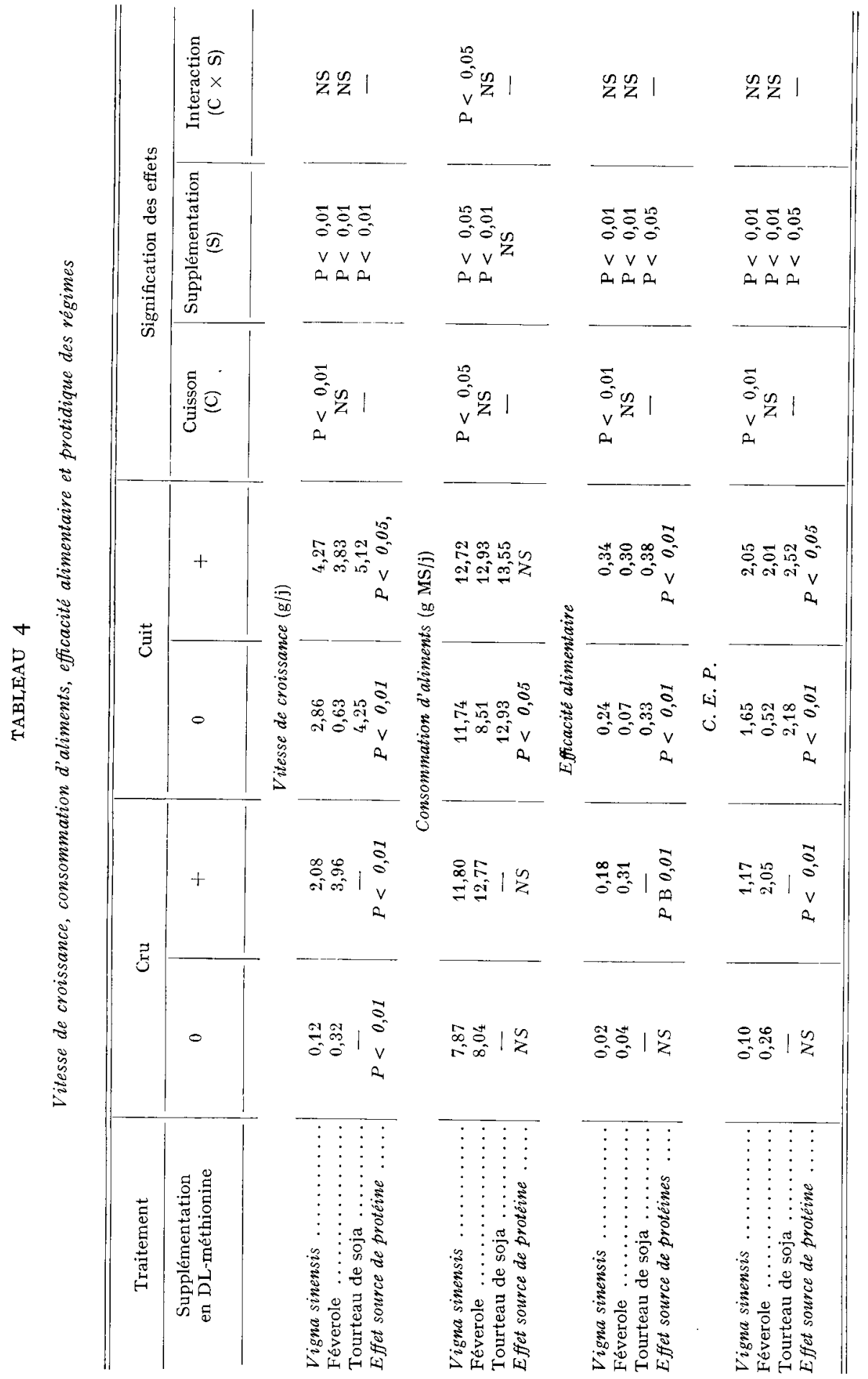


ment l'utilisation digestive apparente des matières azotées des régimes à base de Vigna ( + I3 p. I0o, $\mathrm{P}<0,0 \mathrm{I}$ ) et à un moindre degré, celles des matières azotées de la Féverole $(+4$ p. roo, $\mathrm{P}<0,05)$.

TABLEAU 5

Coefficients d'utilisation digestive apparente des éléments du régime

\begin{tabular}{|c|c|c|c|}
\hline Traitement & Cru & Cuit & Effet cuisson \\
\hline & \multicolumn{2}{|c|}{ Matière organique } & \\
\hline \multirow[t]{2}{*}{$\begin{array}{l}\text { «Vigna sinensis } \text {. . . . . . } \\
\text { Féverole .............. } \\
\text { Tourteau de soja...... } \\
\text { Effet source de protéine }\end{array}$} & $\begin{array}{c}86,9 \pm 0,3\left({ }^{1}\right) \\
89,4 \pm 0,4 \\
= \\
P \pm 0,05\end{array}$ & $\begin{array}{l}88,5 \pm 0,5 \\
90,5 \pm 0,3 \\
93,8 \pm 0,9 \\
P \pm 0,05\end{array}$ & $\begin{array}{c}\mathrm{P}<0,05 \\
\mathrm{P}<0,05 \\
\quad-\end{array}$ \\
\hline & \multicolumn{2}{|c|}{ Azote } & \\
\hline $\begin{array}{l}\text { "Vigna sinensis" ....... } \\
\text { Féverole } \ldots . . . \ldots \ldots \ldots \\
\text { Tourteau de soja....... } \\
\text { Effet source de protéine }\end{array}$ & $\begin{aligned} 70,7 & \pm 1,3 \\
83,5 & \pm 1,2 \\
& - \\
P & <0,01\end{aligned}$ & $\begin{array}{c}80,1 \pm 2,0 \\
87,0 \pm 1,1 \\
87,3 \pm 1,1 \\
P<0,01\end{array}$ & $\begin{array}{c}\mathrm{P}<0,01 \\
\mathrm{P}<0,05 \\
\quad-\end{array}$ \\
\hline
\end{tabular}

(1) Entre parenthèses : écart-type $s_{\bar{x}}^{\bar{x}}$.

Par ailleurs, quel que soit le traitement, les digestibilités apparentes de la matière organique et surtout de l'azote des régimes à base de Vigna sont inférieures respectivement à celles des régimes à base de Féverole et de tourteau de soja. En revanche, l'utilisation digestive des matières azotées de la Féverole cuite est équivalente à celle du tourteau de Soja.

\section{C. - Composition corporelle, efficacité de la rétention azotée}

Les résultats de la composition corporelle figurent au tableau 6. La cuisson a tendance à augmenter la teneur en lipides chez les animaux recevant les régimes à base de Féverole ( +9 p. IOo) et surtout de Vigna ( 27 p. IOO). Le pourcentage de protéines, par contre, reste à peu près constant quel que soit le régime.

L'efficacité de la rétention azotée (CUP) est nettement améliorée à la fois par la supplémentation et la cuisson pour les régimes à base de Vigna, par la supplémentation seule, et à un degré moindre (5 p. Ioo) par la cuisson, pour les régimes à base de Féverole. Les CUP des régimes à base de Vigna cuit et supplémenté et de Féverole crue supplémentée, représentent respectivement 9 I et 80 p. roo de celui obtenu avec le régime à base de tourteau de soja non supplémenté. 


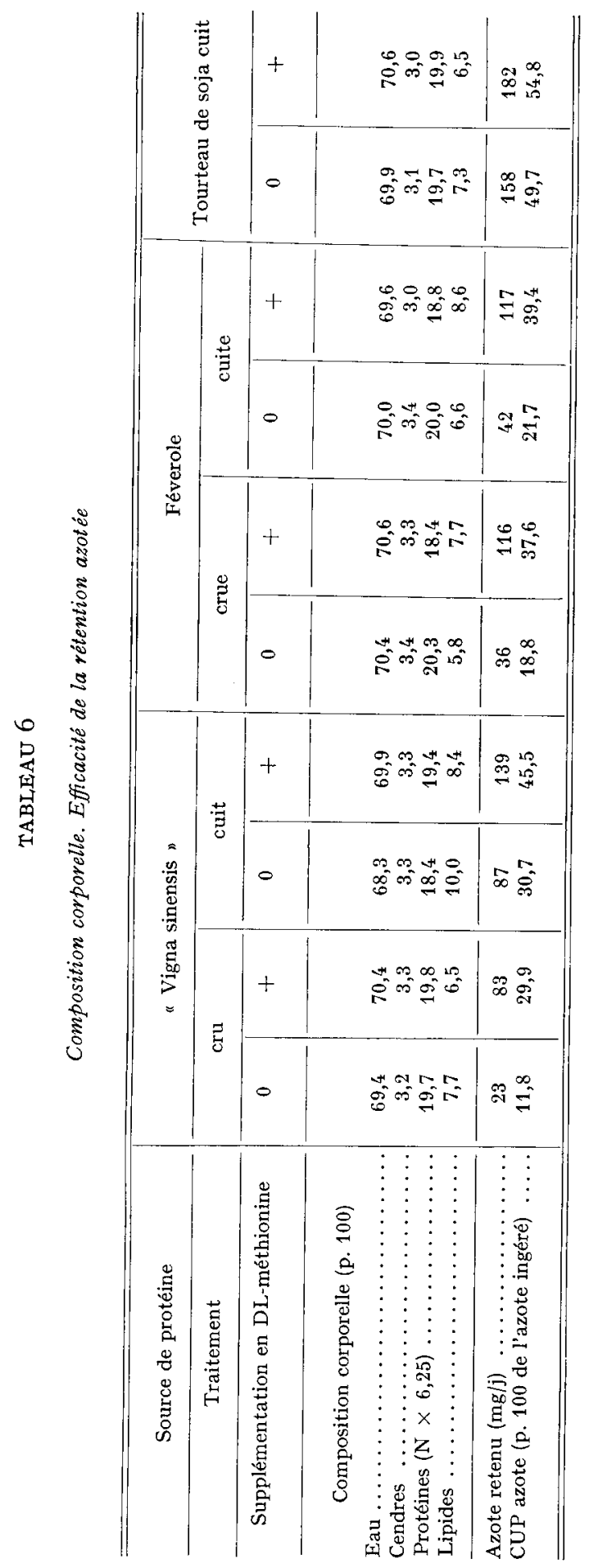




\section{III. - DISCUSSION}

L'amélioration de la vitesse de croissance observée à la suite de la supplémentation des graines de Vigna et de Féverole en DL-méthionine confirme les observations déjà anciennes de FInks, Jones et Johns, (I922) et celles plus récentes de Sherwood, Weldon et Peterson (I954) ou de Wilson et McNab, (I972). Les nombreuses analyses chimiques des protéines de légumineuses (ADRIAN, I963; Pion, De Beisunce et Fauconneau, I963; Evans et Bandmer, i967 ; OwusuDOMFed, Christensen et OWEN, I970) font apparaître en effet, un déficit en acides aminés soufrés de $40 \mathrm{p}$. Ioo pour le régime à base de Vigna, $30 \mathrm{p}$. Ioo pour le régime à base de Féverole et de Io p. Ioo pour le tourteau de soja par rapport aux normes définies par RÉRAT et HENRY (I965). L'amélioration de la vitesse de croissance pondérale ainsi que de la quantité d'aliment consommée, consécutive à la supplémentation s'accompagne surtout d'une amélioration de l'efficacité alimentaire et azotée, en accord avec les résultats classiquement connus.

Le traitement hydrothermique du Vigna entraîne à la fois une amélioration de la vitesse de croissance et de l'utilisation digestive et métabolique de l'azote (Borchers et Ackerson, I950 ; Evans et Bandmer, I967 ; Mescle et Pion, I97I), et l'on peut penser à une destruction au cours de cette opération de substances thermolabiles inhibitrices de la digestion des protéines. Ces résultats ont été par ailleurs confirmés par SEvilla-Fusebio et al., (I968) chez le Porc alimenté à base de graines de Vigna; il est toutefois nécessaire, de limiter la durée du traitement (ADRIAN, I963 ; TANNOUS et ULLAH, I969), veiller à la température de cuisson (VERMOREL, I973), et nuancer les résultats suivant la variété de Vigna utilisée.

Le traitement thermique pratiqué ne semble pas avoir d'effet bénéfique sur la croissance des animaux recevant de la Féverole, ainsi que l'a signalé NiTSAN (I97I) chez le Rat, en contradiction cependant avec des résultats obtenus sur le Rat et sur le Poulet par Borchers et ACKERSON (I950), Wilson et MCNAB (I972). L'amélioration de la digestibilité des protéines observée dans notre expérience à la suite de l'autoclavage tendrait à confirmer l'existence de substances inhibitrices des enzymes protéolytiques, en accord avec NITSAN, (I97I) et WILSON, MCNAB et BENTLEY (I972). L'infériorité des résultats de croissance obtenus avec des régimes à base de Féverole par rapport aux régimes renfermant le tourteau de soja, peut encore s'expliquer par l'importance des téguments cellulosiques ou des tanins dans la graine, hypothèse déjà évoquée par Henry (I970), Nitsan (I97I) et HENRY et Bourdon (I973).

En conclusion, on peut penser, à la lumière de nos résultats obtenus sur le Rat pendant une durée limitée, que les graines de Vigna Sinensis autoclavées à I20 ${ }^{\circ}$ pendant I 5 minutes et supplémentées à raison de 0,15 p. Ioo en DL-méthionine constituent, pour l'alimentation animale, une source azotée de valeur comparable au tourteau de soja cuit. 


\title{
SUMMARY
}

\author{
PROTEIN VALUE OF COWPEAS (VIGNA SINENSIS) \\ AND FIEL, BEANS (VICIA FABA) AS COMPARED TO SOYBEAN OIL-MEAL, \\ FOR THE RAT
}

The protein value of raw heated Vigna Sinensis seeds supplemented or not by synthetic DL-methionine and Vicia faba beans (var. Ascott) was compared with that of heated soybean oil-meal supplemented or not by synthetic DL-methionine when fed at 14 p. Ioo protein level to rats during i 2 days.

The criteria chosen were : growth rate, protein efficiency ratio (P. E. R.), N digestibility, and $\mathrm{N}$ retention estimated from analysis of carcass.

Supplementation significantly improved the nutritive value of both Vigna Sinensis seeds $(\mathrm{P}<0.0 \mathrm{I})$ Vicia faba bean $(\mathrm{P}<0.01)$ and soybean oil-meal $(\mathrm{P}<0.05)$.

Heating significantly improved $(\mathrm{P}<0.0 \mathrm{I})$ the nutritive value of Vigna Sinensis seeds. The effects of supplementation and heating on Vigna Sinensis seeds were additive on growth rate, P. E. R. and nitrogen retention. In another connection, heating only improved $\mathrm{N}$ digestibility $(\mathrm{P}<0.05)$ of Vicia faba beans.

\section{RÉFÉRENCES BIBLIOGRAPHIQUES}

Adrian J., Ig63. Étude de la valeur protidique de trois légumineuses domestiques africaines (Voandzou, Dolique et Niébé). Ann. Nutr. Alim., 17, I-18.

Aikroyd W. R., Doughty J., I964. Legumes in Human Nutrition. Nutritional Studies $\mathrm{n}^{\mathbf{0}}$ I9, Rome F. A. O.

Borchers R., Ackerson C. M., 1950. The nutritive value of legumes seeds. Effect of autoclaving and the trypsin inhibitor test for I7 species. J. Nutr. 41, 339-345.

Busson F., 1965. Plantes alimentaires de l'Ouest africain. Thèse doc. Fac. Sci. Marseille.

DerieuX M., rg69. Résultats d'essais préliminaires à l'étude de deux légumineuses vivrières. Proc. VIIth Annual Meeting C. F. C. S. Martinique-Guadeloupe 164-172.

Evans R. J., Bandmer S. L., I967. Nutritional value of legume seed proteins. J. Agric. Food Chem., 14, 439-443.

Finks A. J., Jones D. B., Johns C. O., 1922. The role of cystine in the dietary properties of the proteins of the cowpeas Vigna Sinensis, and of the field peas, Pisum sativum. J. Biol. Chem., 52, 4034 IO.

Henry Y., i97o. La Féverole dans l'alimentation du Porc. Bull. Tech. Inf., 253, 625-634.

Henry Y., Bourdon D., I973. Utilisation digestive de l'énergie et des matières azotées de la Féverole, sous forme entière ou décortiquée, en comparaison avec le tourteau de soja. Journées Rech. Porcine en France, Paris, I. N. R. A. I. T. P. éd., ro5-I r4.

Liener I. D., Pallansch M. J., I962. Toxic factors in edible legumes and their elimination. Amer. J. Clin. Nutr., 11, 28I-285.

Mescle J. F., Pion R., I97I. Participation à l'étude de la valeur nutritive des protéines du Vigna Sinensis (données non publiées).

Osborne T. B., Mendel L. B., 1917. The use of soyabean as food. J. Biol. Chem. 32, 369.

Owusu-Domfed K., Christensen D. A., Owen B. D., x97o. Nutritive value of some Ghanaian feedstuffs. Can. J. Anim. Sci., 40, I-4I.

Morisson F. B., I967. Feeds and feeding 22nd éd. Morisson Publishing Co, Ithaca, New York.

Nitsan S., I97I. Vicia faba beans vs. Soyabean meal as a source of protein. J. Sci. Fd. Agric., 22, $252-255$.

Pion R., De Belsunce C., Fauconneau G., ig63. Composition en acides aminés de quelques aliments. Ann. Biol. anim. Bioch. Biophys., 3 (n H. S.), I I-I8.

Pointel J. G., I967. Contribution à la conservation du Niébé. Agron. trop., 10, 925-932.

Rérat A., Henry Y., r965. Détermination des besoins en acides aminés soufrés chez le Rat blanc en croissance. Ann. Biol. anim. Bioch. Biophys., 5, 469-48I. 
Sherwood F. W., Weldon V., Peterson W. J., I954. Effect of cooking and of methionine supplementation on the growth promoting property of cowpea Vigna Sinensis protein. J. Nutr., 52, I99 208.

Sevilla-Eusebio J., Gonzales R. R., Eusebio J. A., Alcantara P. F., ig68. Effect of heat on the biological value of Munggo, Paayap, Tapilan and Kadyox beans. Philippine Agriculturist, 52, 216-232.

TAnnous R. I., UlLAH M., I969. Effects of autoclaving on nutritional factors in legume seeds. Trop. Agric. Trin., 46, 123-г29.

Thivend P., Mercier Christiane, Guilbot A., I965. Dosage de l'amidon dans les milieux complexes. Ann. Biol. anim. Bioch. Biophys, 5, 513-526.

VERMOREL, I973. Communication personnelle.

Wilson B. J., McNab J. M., Bentley H., 1972. Trypsin inhibitor activity in the field bean (Vicia faba L.). J. Sci. of Agric., 23, 679-684.

Wilson F. W., MCNAB J. M., 1972. The effect of autoclaving and methionine supplementation on the growth of chicks given diets containing field beans (Vicia Faba L.). Br. Poult. Sci., 13, 67-74. 